\title{
Retrievable hydrogels for ovarian follicle transplantation and oocyte collection
}

\author{
Peter D. Rios ${ }^{1,2,3}$ | Ekaterina Kniazeva ${ }^{3}$ | Hoi Chang Lee ${ }^{3}$ | Shuo Xiao ${ }^{3}$ | \\ Robert S. Oakes ${ }^{4}$ | Eiji Saito ${ }^{4}$ | Jacqueline S. Jeruss ${ }^{4,5,6}$ | Ariella Shikanov ${ }^{4,6}$ \\ Teresa K. Woodruff ${ }^{2,3}$ | Lonnie D. Shea ${ }^{4,6,7}$
}

${ }^{1}$ Department of Biomedical Engineering, Northwestern University, Evanston, Illinois

${ }^{2}$ Robert H. Lurie Comprehensive Cancer Center, Northwestern University, Chicago, Illinois

${ }^{3}$ Department of Obstetrics and Gynecology, Northwestern University, Chicago, Illinois

${ }^{4}$ Department of Biomedical Engineering, University of Michigan, Ann Arbor, Michigan

${ }^{5}$ Department of Surgery, University of Michigan, Ann Arbor, Michigan

${ }^{6}$ University of Michigan Comprehensive Cancer Center, Ann Arbor, Michigan

${ }^{7}$ Department of Chemical Engineering, University of Michigan, Ann Arbor, Michigan

\section{Correspondence}

Lonnie D. Shea, Ph.D., Department of Biomedical Engineering, University of Michigan, 1119 Carl A. Gerstacker Building, 2200 Bonisteel Boulevard, Ann Arbor, MI 48109-2099, USA.

Email: Idshea@umich.edu

Funding information

National Institutes of Health (NIH) National Center for Translational Research in Reproduction and Infertility (NCTRI), Grant/ Award Number: P5OHD076188

\begin{abstract}
Cancer survivorship rates have drastically increased due to improved efficacy of oncologic treatments. Consequently, clinical concerns have shifted from solely focusing on survival to quality of life, with fertility preservation as an important consideration. Among fertility preservation strategies for female patients, ovarian tissue cryopreservation and subsequent reimplantation has been the only clinical option available to cancer survivors with cryopreserved tissue. However, follicle atresia after transplantation and risk of reintroducing malignant cells have prevented this procedure from becoming widely adopted in clinics. Herein, we investigated the encapsulation of ovarian follicles in alginate hydrogels that isolate the graft from the host, yet allows for maturation after transplantation at a heterotopic (i.e., subcutaneous) site, a process we termed in vivo follicle maturation. Survival of multiple follicle populations was confirmed via histology, with the notable development of the antral follicles. Collected oocytes (63\%) exhibited polar body extrusion and were fertilized by intracytoplasmic sperm injection and standard in vitro fertilization procedures. Successfully fertilized oocytes developed to the pronucleus (14\%), two-cell (36\%), and four-cell (7\%) stages. Furthermore, ovarian follicles cotransplanted with metastatic breast cancer cells within the hydrogels allowed for retrieval of the follicles, and no mice developed tumors after removal of the implant, confirming that the hydrogel prevented seeding of disease within the host. Collectively, these findings demonstrate a viable option for safe use of potentially cancer-laden ovarian donor tissue for in vivo follicle maturation within a retrievable hydrogel and subsequent oocyte collection. Ultimately, this technology may provide novel options to preserve fertility for young female patients with cancer.
\end{abstract}

\section{KEYWORDS}

alginate, biomaterial, follicle, hydrogel, oocyte

\section{1 | INTRODUCTION}

Advances in chemo- and radiotherapy have significantly improved cancer survivorship rates worldwide. As of 2016, the American Cancer Society (ACS) estimates that more than 15 million individuals in the
United States are currently in remission. By 2024, ACS projects this number will increase to more than 19 million, of which 9 million will be female (American Cancer Society, 2014; 2017). In particular, the 5-year survival rate for pediatric patients (0- to 14-year age group) has improved to $87 \%$ during the 2008-2012 period (Wallace, Kelsey, \& 
Anderson, 2016). Given these estimates, clinical concerns have shifted to include fertility preservation after treatment.

Alkylating chemotherapy or irradiation to the pelvis or abdomen in female patients can be gonadotoxic and cause irreversible damage to the ovaries, reducing the patient's ability to conceive successfully by $50 \%$ (Salama \& Woodruff, 2015; Shea, Woodruff, \& Shikanov, 2014). In cases where doses of abdominal radiation of 20-30 Gy are used, the risk of adverse pregnancy outcomes can be as high as $90 \%$ (Kim, Kim, Lee, \& Woodruff, 2016; Wallace, Shalet, Hendry, MorrisJones, \& Gattamaneni, 1989; Wo \& Viswanathan, 2009). Currently available options to preserve fertility before treatment include embryo cryopreservation, oocyte cryopreservation, and ovarian tissue cryopreservation, though embryo and oocyte cryopreservation may not be applicable to pediatric patients.

The transplantation of cryopreserved ovarian tissue is the only clinical option available to restore fertility using cryopreserved tissue and has resulted in 60 live births reported to date (Donnez \& Dolmans, 2015). This procedure does not require hormonal stimulation or a sperm donor, preserves ovarian follicles at all stages of maturation, including primary and primordial follicles, and can be applied to prepubertal girls (Kim et al., 2016; Kondapalli \& Ginsberg, 2012; Meirow, Ra, \& Biderman, 2014). However, this method is still considered experimental and is associated with several challenges related to reimplantation that prevent it from becoming the gold standard for fertility preservation. These challenges include ischemic injury early after transplantation due to insufficient tissue revascularization, which significantly reduces the ovarian follicle pool, follicular atresia, and risk of reseeding malignant cancer cells (Demeestere, Simon, Emiliani, Delbaere, \& Englert, 2009; Donnez, Squifflet, \& Dolmans, 2009; Salama \& Woodruff, 2015). In particular, the reintroduction of malignant disease remains a primary concern as $12.4 \%$ of patients died due to recurrence after reimplantation of cryopreserved ovarian tissue according to a 12-year study (Imbert et al., 2014). Thus, the presence of cancer cells in cryopreserved ovarian tissue (Abir et al., 2014; Dolmans, Luyckx, Donnez, Andersen, \& Greve, 2013; Rosendahl, Greve, \& Andersen, 2013) has motivated strategies such as follicle isolation and transplantation as a means to reduce or remove the cancer cell population (Kniazeva et al., 2015).

Hydrogels have been used for the transplantation of ovarian tissue or isolated follicles as a means to enhance efficacy. The three-dimensional architecture of the hydrogel physically supports the follicles, maintains oocyte-somatic cell connections, and permits expansion of early-stage follicles (Shea et al., 2014). In particular, fibrin hydrogels have been used for ovarian follicle transplantation to facilitate the interaction of the transplant with the host. Transplantation of fibrin-encapsulated follicles has promoted their survival after transplantation, enabled growth and maturation in vivo, and restored endocrine function in the ovariectomized mice (Kniazeva et al., 2015; Luyckx et al., 2014; Smith et al., 2014). Restoration of endocrine function is particularly important and necessary for successful clinical pregnancies (Donnez et al., 2013; Oktay et al., 2001). Fibrin hydrogels modified with vascular endothelial growth factor (VEGF) and transplanted into the orthotropic site of ovarian bursa have improved encapsulated murine follicle function and resulted in live births via natural pregnancy (Kniazeva et al., 2015; Shikanov, Ph et al.,
2011; Smith et al., 2014). There are at least two significant challenges with these approaches however: (i) the live births required a relatively large number of transplanted follicles that may be difficult to obtain in a clinical setting from human tissue, and (ii) the degradation of the fibrin hydrogel and integration of the graft with the host tissue could allow for dissemination of residual cancer cells.

In this report, we investigated a strategy for transplantation of ovarian follicles within nondegradable alginate hydrogels to allow in vivo follicle maturation, with subsequent retrieval of the graft to obtain mature oocytes for in vitro fertilization (IVF). Alginate hydrogels have been used for encapsulation to provide support for culture and maturation of mouse follicles (Filatov, Khramova, \& Semenova, 2014; Hornick, Duncan, Shea, \& Woodruff, 2012; Hornick, Duncan, Shea, \& Woodruff, 2013; Telfer \& Zelinski, 2013; Xiao, Duncan et al., 2015; Xu, Banc, Woodruff, \& Shea, 2009) and human follicles (Laronda et al., 2014; Xiao, Zhang et al., 2015) in vitro. Oocytes can be retrieved from alginate-encapsulated murine follicles for IVF and subsequently implanted to produce healthy offspring (Xu, Kreeger, Shea, \& Woodruff, 2006). Here, we proposed to apply the alginate hydrogels for in vivo maturation at a subcutaneous site, with the hydrogels providing support for follicle growth, while also presenting an effective barrier that limits integration with the host tissue and potentially facilitates implant retrieval. After encapsulation and transplantation, hydrogels were extracted and carefully dissected to collect oocytes for in vitro maturation (IVM) and subsequent fertilization by intracytoplasmic injection and standard IVF. Furthermore, we assessed the ability of cancer cells encapsulated together with the follicles to initiate tumor development within the transplant recipient. We were able to demonstrate that retrievable hydrogels used for follicle maturation provide a unique opportunity to maintain follicle architecture and allow maturation within the in vivo environment, while eliminating the risks associated with potentially cancer-laden ovarian tissue.

\section{2 | MATERIALS AND METHODS}

\section{1 | Follicle isolation and hydrogel fabrication}

Ovaries were extracted from 12-day-old C57BL/6j x CBA/Ca female mice (Harlan Laboratories, Indianapolis, IN) and mechanically dissected using insulin syringe needles (1cc, 28G ${ }^{1 / 2}$ ) (BD Biosciences, Franklin Lakes, NJ). The tissue is dissected as a means to collect follicles primarily and reduce the population of stromal cells. Although stromal cells are present, their quantity has been reduced. The resulting ovarian fragments, approximately $300 \mu \mathrm{m}$ in diameter, were allowed to aggregate in a 0.6-ml Eppendorf tube in Leibovitz's L-15 media (Gibco, Carlsbad, CA) supplemented with $10 \%$ fetal bovine serum (FBS; Gibco) and 1\% PenStrep (Sigma-Aldrich, St. Louis, MO). Follicle isolation was performed on a heated stage at $37^{\circ} \mathrm{C}$ in a sterilized hood to prevent contamination. Either three, two, or one ovary was dissected and incorporated into each of the gels, which correspond to a mixture of primordial, primary, and secondary follicles with approximately 1,100, 730, or 360 follicles, respectively, based on follicle quantification from native 12-day-old ovaries. To form alginate 
hydrogels, the supernatant was removed until only the follicle aggregate remained. Next, $7 \mu \mathrm{l}$ of $0.5 \%$ alginate (NovaMatrix, Sandvika, Norway) mixed with sterile phosphate buffered saline (PBS-/-) (Perkin Elmer, Waltham, MA) was slowly added to the tube without disturbing the follicles, followed by $50 \mu \mathrm{l}$ of thrombin/ $\mathrm{Ca}^{2+}$ (SigmaAldrich, St. Louis, MO), and the resulting mixture was allowed to crosslink for $3 \mathrm{~min}$. The thrombin/ $\mathrm{Ca}^{2+}$ solution was created by combining $50 \mathrm{IU} / \mathrm{ml}$ thrombin with $40 \mathrm{mM} \mathrm{CaCl}_{2}$. Hydrogels were approximately $3 \mathrm{~mm}$ in diameter and kept in L-15 media before transplantation.

\section{2 | Ovariectomy and transplantation}

Hydrogels were transplanted into C57BL/6j $\times$ CBA/Ca adult 6- to 7-week-old female mice after ovariectomy, either into the ovarian bursa or subcutaneously in the dorsal region. Mice were anesthetized with intraperitoneal injection of $100 \mathrm{mg} / \mathrm{kg}$ of ketamine and $15 \mathrm{mg} / \mathrm{kg}$ of xylazine. Hydrogels were implanted into the bursa according to a previous report (Kniazeva et al., 2015). For subcutaneous implants, a small incision was made on the back to form a pocket, hydrogels were inserted, and then the incision was closed using a 5-0 vicryl suture (Ethicon, Somerville, NJ). Sterile surgical procedures, postoperative procedures, and daily care were performed according to protocols approved by the Northwestern University Institutional Animal Care and Use Committee.

\section{3 | Retrieval and analysis of hydrogel transplants}

After 7 days, mice were euthanized, and ovarian bursae or subcutaneous implants were retrieved for histological analysis. On removal, samples were fixed in $4 \%$ paraformaldehyde, dehydrated in $70 \%$ ethanol, paraffin-embedded, serial-sectioned, and stained with hematoxylin and eosin (H\&E). Follicle counts were performed by an experienced researcher blinded to experimental conditions. Follicles were quantified according to the following classification scheme: primordial follicles contained four to six squamous granulosa cells, primary follicles were enclosed by and contained a mix of squamous and cuboidal cells, secondary follicles contained two layers of cuboidal granulosa cells, multilayered secondary follicles contained more than two layers of cuboidal layers with no presence of a cavity (i.e., corpus luteum), and antral follicles were identified by the presence of an oocyte surrounded by several layers of cuboidal cells and a defined corpus luteum.

\section{4 | Oocyte collection, IVM assay, and immunofluorescence}

Hydrogels were explanted 7 days after transplant in the bursa or subcutaneous site and placed in L-15 medium. Hydrogel samples were mechanically dissected on a heated-stage microscope using insulin syringe needles $\left(1 \mathrm{cc}, 28 \mathrm{G}^{1 / 2}\right)$ to obtain antral follicles. Antral follicles were carefully punctured to release oocytes into the surrounding media. Only oocytes with a visible germinal vesicle, and thus arrested at prophase I, were transferred to IVM media alpha minimum essential medium ( $\alpha$-MEM) with 10\% FBS, $1.5 \mathrm{IU} / \mathrm{ml}$ human chorionic gonado- tropin, and $10 \mathrm{ng} / \mathrm{ml}$ epidermal growth factor (BD Biosciences, Franklin Lakes, NJ). Oocytes were incubated for $16 \mathrm{hr}$ at $37^{\circ} \mathrm{C}$ in $5 \% \mathrm{CO}_{2}$ and then imaged to confirm MII status, denoted by polar body extrusion. A subset of metaphase II (MII) oocytes derived from hydrogel samples from the bursa or subcutaneous site was immunofluorescently stained and imaged with a confocal microscope (Leica Microsystem) using previously cited methods (Xiao, Zhang et al., 2015) to assess the morphology of the meiotic spindle, a marker of egg quality.

\subsection{IVF and embryo development}

Oocytes derived from subcutaneously transplanted hydrogel samples containing 1,100 follicles were used for IVF and embryo development studies. After confirmation of MII status via polar body extrusion after IVM, oocytes were fertilized with sperm collected from the epididymis of 8- to 10-week-old C57BL/6j x CBA/Ca male mice. Isolated epididymides were placed in a $1.5-\mathrm{ml}$ Eppendorf tube with $1 \mathrm{ml}$ of human tubular fluid (HTF) medium supplemented with $0.4 \%$ of bovine serum albumin. Sperm were incubated for $30 \mathrm{~min}$ at $37^{\circ} \mathrm{C}$, and the supernatant layer containing healthy and motile sperm was collected. Sperm was either injected directly into oocytes using intracytoplasmic sperm injection (ICSI) or placed in the HTF medium with oocytes using the standard IVF procedures. For ICSI, MII oocytes were placed in $50 \mu \mathrm{l}$ drops of EmbryoMax KSOM Medium ( $1 \times$ with $1 / 2$ amino acids; Millipore, Burlington, MA) on a heated microscope stage (Nikon Eclipse TE300), and sperm were microinjected using a Piezo-drill tip (Eppendorf, Hamburg, Germany) containing Fluorinert FC770 (Sigma-Aldrich, St. Louis, $\mathrm{MO}$ ) to generate a pulse sufficient to penetrate the zona pellucida. After sperm injection, MII oocytes were left in $50 \mu$ of KSOM medium submersed in Embryo Culture Oil (Irvine Scientific, Santa Ana, CA) and monitored for up to $96 \mathrm{hr}$ to evaluate embryo outcomes. Standard IVF was also used to fertilize MII oocytes. In brief, the zona pellucida was denuded using acetic acid and transferred to HTF medium. A low sperm concentration of 5,000 sperm total was added to $1 \mathrm{ml}$ of HTF with MII oocytes for $24 \mathrm{hr}$ and then transferred to a fresh $50 \mathrm{\mu l}$ of KSOM medium without sperm. MII oocytes were then cultured for another $72 \mathrm{hr}$ for a total of $96 \mathrm{hr}$ to assess embryo outcomes after fertilization.

\section{6 | Cancer-cell-laden hydrogel transplants, in vivo luminescence imaging, and organ histology}

Alginate hydrogel $(7 \mu \mathrm{l}, 0.5 \%)$ containing approximately 200 triple negative breast cancer cell line (MDA-MB-231 BR) cells expressing luciferase and approximately 360 ovarian follicles from a cluster of differentiation-1 (CD-1) female mouse donor was transplanted into the dorsal subcutaneous site of non-obese diabetic-severe combined immunodeficiency (NOD-SCID) gamma (NSG) mice. At Day 7, mice were injected $(150 \mathrm{mg} / \mathrm{kg})$ with D-luciferin firefly $(20 \mathrm{mg} / \mathrm{ml}$ in sterile PBS) and imaged 10 min after injection on an in vivo imaging system (IVIS; Perkin Elmer Xenogen IVIS Spectrum Bioluminescence System) to confirm the presence of cancer cells in the gel. Hydrogel implants were removed after imaging, and mice were monitored for 3 weeks after removal of the gel, at which time the mice were imaged again to determine if cancer cells were present at the 


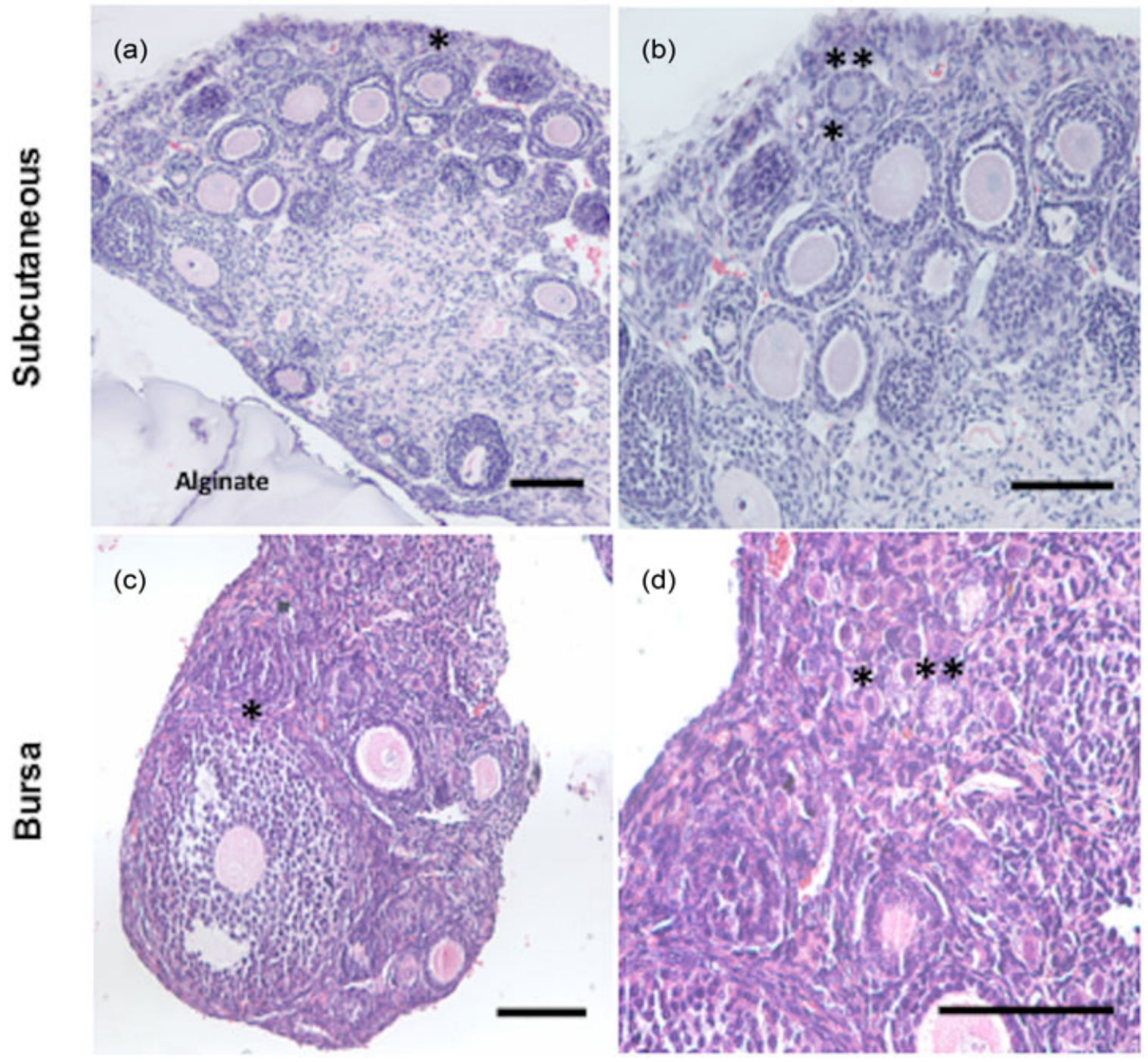

FIGURE 1 Follicle survival and growth in hydrogel explants 7 days after transplantation in subcutaneous and bursa sites. Antral, multilayered secondary, and secondary follicles were observed in extracted ovarian grafts from the subcutaneous and bursa sites (a, b and c, d). In panel (a), surrounding alginate material served to maintain the separation of host tissue from follicles transplanted subcutaneously. A representative antral follicle is denoted with an asterisk $\left(^{*}\right)$ in panels (a) and (c). Large numbers of primordial and primary follicles were identified in all extracted hydrogels and denoted with a single $\left({ }^{*}\right)$ or double asterisk $\left(^{* *}\right)$, respectively, in panels (b) and (d). Hydrogel implant in panels (a)-(b) and (c)-(d) contained approximately 360 and 1,100 follicles, respectively. Similar results were observed for alginate hydrogels with follicle populations of approximately 730 ovarian follicles at both sites. Scale bar: $100 \mu \mathrm{m}$ [Color figure can be viewed at wileyonlinelibrary.com]

subcutaneous transplant site. Liver and lungs were excised from all recipients within a week after the last imaging time point, fixed in $4 \%$ paraformaldehyde (PFA), paraffin-embedded, and stained with $H \& E$ to assess if metastasis occurred from the initial transplant site.

\section{3 | RESULTS}

\section{1 | In vivo follicle transplantation within hydrogels and histological analysis}

Hydrogel encapsulation was evaluated for its ability to support follicle survival and development in vivo. Specifically, alginate was chosen to encapsulate mechanically isolated follicle aggregates and subsequently transplanted subcutaneously in the dorsal region (a heterotopic site) of ovariectomized female recipient mice, with transplantation into the ovarian bursa (an orthotopic site) used as a control based on the previous reports of follicle maturation and consequent successful live births. A range of follicle quantities $(1,100,730$, or 360$)$ was encapsulated within hydrogels, and surviving populations of follicles at all developmental stages were observed in grafts extracted from both the subcutaneous and bursa transplant sites (Figure 1a-d). Notably, antral follicles containing oocytes were identified within the hydrogel explants (Figure 1a,c), which were not initially present within the encapsulated follicle populations and thus developed from this original pool (Supporting Information Figure 1a-c).

Quantification of follicle populations from removed grafts implanted subcutaneously indicated that a majority of the follicles 
were primordial, with antral follicles observed in all conditions (Figure 2a,b). For the subcutaneous grafts, the greatest number of transplanted follicles resulted in the smallest percentage of surviving follicles (Figure 2c). Transplantation of the smallest initial follicle numbers resulted in the greatest survival percentage ( $\approx 76 \%$ ). Relative to the transplanted population, the number and percentage of primordial follicles had decreased, with a corresponding increase in the number and percentage of primary and secondary follicles, suggesting follicle maturation within the bead during the time of transplantation. The number of recovered follicles from the bursa significantly decreased, and recovery from this site was inefficient relative to the subcutaneous site. Transplantation of the smallest initial numbers of follicles (i.e., 360 follicles) in the bursa had the greatest survival percentage of $20 \%$. As with the subcutaneous site, the largest follicle populations were in primordial and secondary stages. Finally, despite the lower resulting number of primordial and secondary follicles, the number of antral stage follicles was similar to the subcutaneous site.

\section{2 | Hydrogel retrieval, oocyte collection, and IVM studies}

On retrieval, the subcutaneously transplanted hydrogels were used to obtain antral follicles for the investigation of oocyte quality. Alginate hydrogels retained their integrity on extraction and allowed for easy follicle dissection (Figure 3a). For transplantation of the largest initial follicle numbers, a total of 54 oocytes in the germinal vesicle stage were retrieved, transferred to IVM media, and imaged
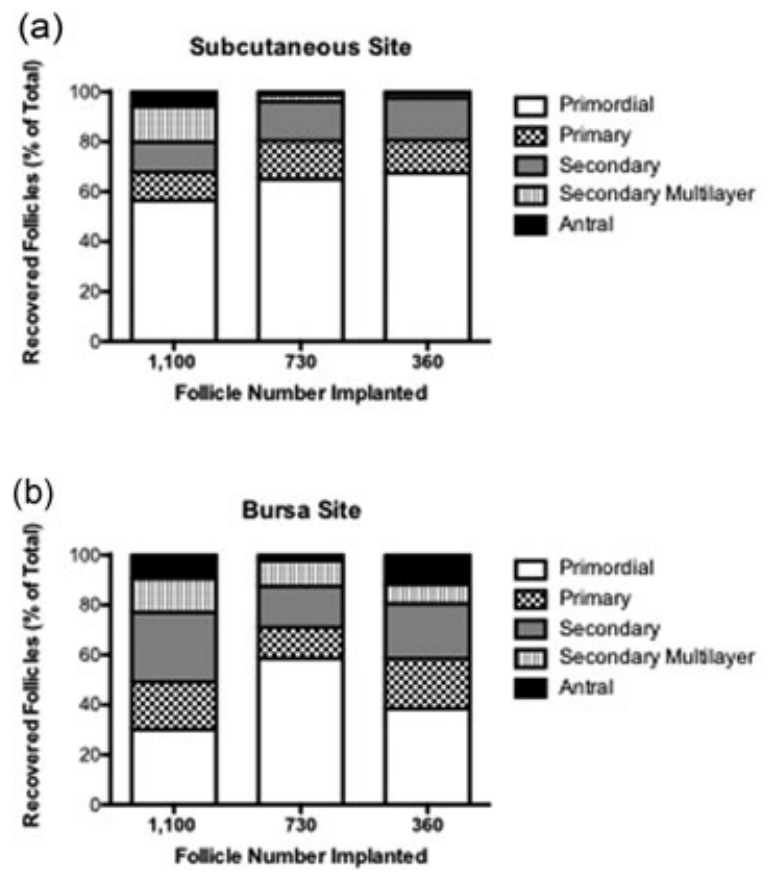

after a 16-hr incubation period. Polar body extrusion was evident in 34 oocytes (MII stage), which corresponds to a $63 \%$ MII rate (Figure 3b,c). For transplants with the reduced number of follicles transplanted, the oocytes had an MII rate of 55\% (730 follicles transplanted) and 46\% (360 follicles transplanted). Importantly, the MII rate was comparable between all three conditions, suggesting that MII oocytes can be achieved with modest numbers of follicles transplanted. Finally, normal spindle morphology was confirmed in MII oocytes derived from alginate hydrogels initially loaded with 1,100 follicles (Figure 3d).

Germinal vesicle oocytes isolated from antral follicles transplanted into the bursa (Figure 4a) underwent IVM to produce an MII rate of $20 \%$ (Figure $4 \mathrm{~b}, \mathrm{c}$ ). Normal spindle morphology indicative of oocyte quality was confirmed in MII eggs after IVM (Figure 4d). Intact hydrogel retrieval from the bursa was more challenging than from the subcutaneous space, which resulted in a lower yield. Subsequent studies thus focused only on the subcutaneous site.

\subsection{Fertilization and embryonic development}

The fertilization competency of the MII stage oocytes that were obtained from the subcutaneously transplanted follicles were subsequently investigated (Figure 5a). ICSI was used to fertilize eggs, and embryo development was monitored for $96 \mathrm{hr}$ after ICSI. Of the $14 \mathrm{MIl}$ eggs that were successfully injected with sperm, oocytes progressed to the pronucleus (14\%), two-cell (36\%), and four-cell (7\%) stages, whereas $40 \%$ of oocytes remained in MII arrest (Figure $5 b$ ). Embryos fertilized via standard IVF progressed to the

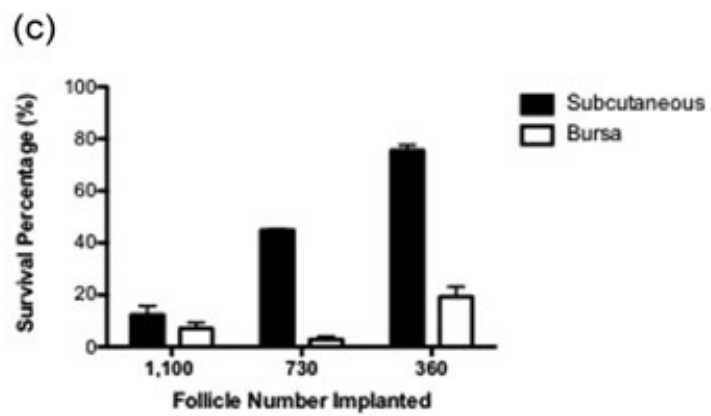

FIGURE 2 Percentage of follicle populations recovered from alginate hydrogel explants 7 days after transplant. Follicle populations (primordial, primary, secondary, multilayered secondary, and antral) were quantified and displayed as a percentage of recovered follicles for the 1,100-, 730-, and 360-follicle implant conditions for the (a) subcutaneous ( $n=3 /$ follicle condition) and (b) bursa transplant sites ( $n=4$ for 1,100-follicle and 730-follicle condition, $n=3$ for 360-follicle condition). (c) Percentage of surviving follicles for the subcutaneous and bursa transplant sites ( \pm standard error of the mean) 

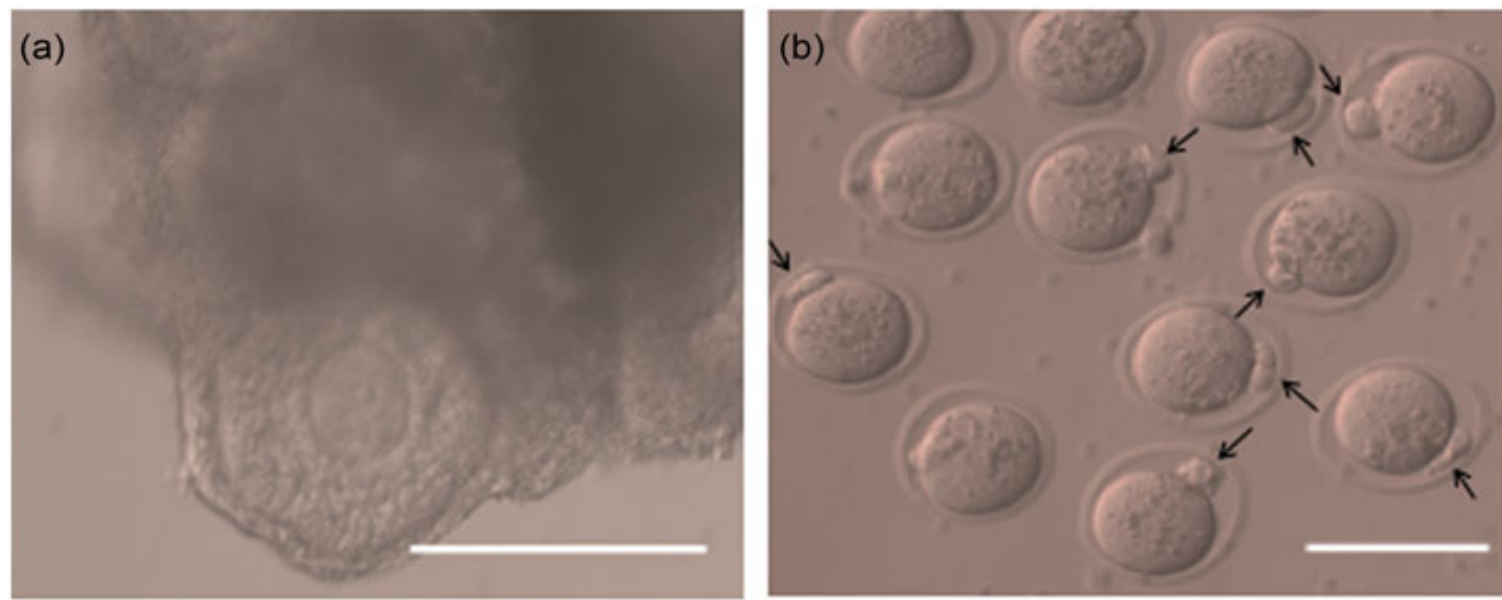

(c)

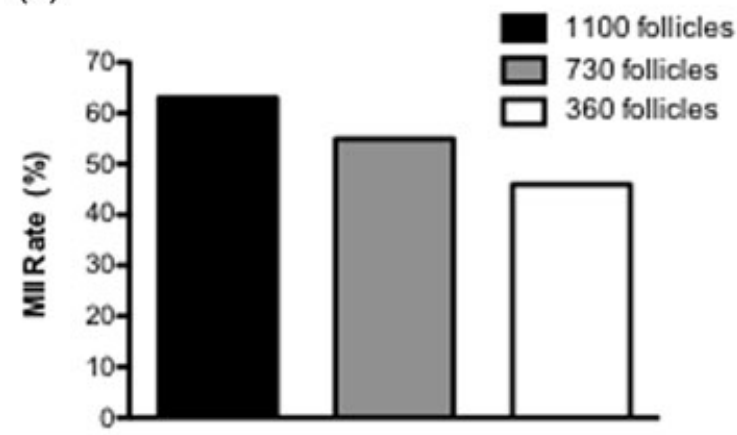

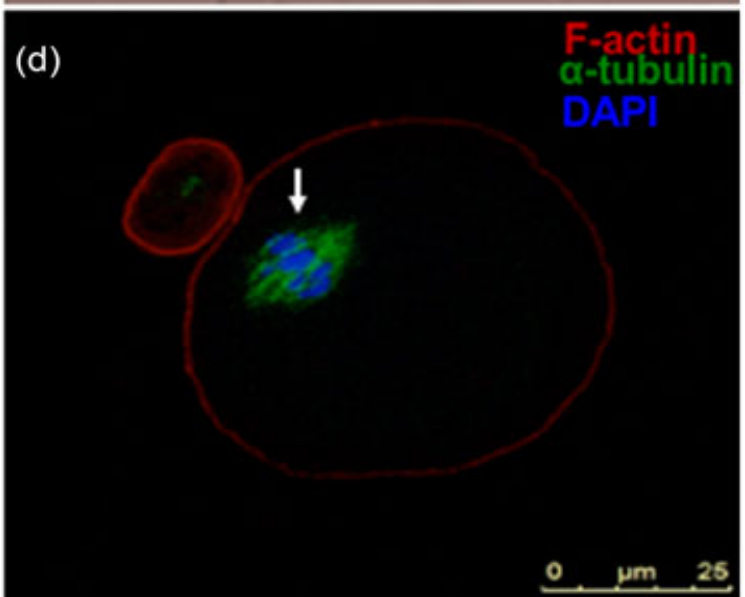

FIGURE 3 Egg retrieval from encapsulated follicles in alginate hydrogels in subcutaneous site 7 days after transplant and their meiotic maturation in vitro. (a) Follicles were easily identified in explanted hydrogels. (b) MIl oocytes were confirmed via polar body extrusion (indicated with black arrows). (c) Egg retrieval and MII status from the subcutaneous site. Of the GV oocytes collected from antral follicles in Day 7 explants with 1,100 ovarian follicles, 34 of 54 oocytes were MII after in vitro maturation (63\% MII rate) from three trials. Oocytes collected from Day 7 explants with 730 or 360 ovarian follicles resulted in an MII rate of 55\% (6 MII oocytes/11 GV oocytes) and $46 \%$ (10 MII oocytes/22 GV oocytes), respectively (d) Normal spindle morphology (indicated by an arrow) was also confirmed in subcutaneously matured oocytes. Image (d) depicts an oocyte obtained from an explant containing ovarian follicles from three ovaries. Scale bar: 100 $\mu$ m (a, b). DAPI, 4',6-diamidino2-phenylindole; GV, germinal vesicle; MII, metaphase II [Color figure can be viewed at wileyonlinelibrary.com]

pronucleus (20\%) and two-cell (40\%) stages, whereas $40 \%$ of oocytes remained in MII arrest (Figure $5 \mathrm{c}$ ).

\section{4 | Cancer-laden hydrogel implants and in vivo imaging}

We next investigated the safety of this strategy by the addition of cancer cells to the isolated follicles followed by their encapsulation and transplantation. We hypothesized that the alginate hydrogel, which prevents direct contact between the host and transplanted tissue, would prevent the escape of cancer cells into the host tissue after transplantation and during retrieval. Approximately 200 MDA-MD-231 BR cells expressing luciferase were encapsulated with approximately 360 ovarian follicles into alginate hydrogels and implanted subcutaneously into NSG mice. The delivery of 20 cells or more of the MDAMD-231 BR cells can lead to tumor formation in this model (Supporting Information Figure 2a-d). In vivo bioluminescence imaging of the follicle/ cancer cell transplants at Day 7 confirmed the presence of cancer cells within the implant $(n=4)$, whereas a luminescence signal was not detected in the follicle transplant-only control group ( $n=4$; Figure 6a). Hydrogels were then removed, and recipient mice were imaged again at 3 weeks after removal of the gel, with no detection of a bioluminescence signal (Figure 6b). The lung and liver were removed within 1 week after imaging and analyzed for metastases. Histology confirmed no metastatic lesions in the lungs or liver compared with control organs in all recipient mice, supporting the safety of this strategy (Figure 7a-d).

\section{4 | DISCUSSION}

This report presents a strategy for in vivo maturation of ovarian follicles, which involved the isolation and encapsulation of follicles into alginate hydrogels, transplantation into the subcutaneous space, and their subsequent retrieval for recovery of antral follicles containing mature oocytes. Alginate hydrogels have been widely used for in vitro follicle culture (Brito et al., 2014; Camboni et al., 2013; Hornick et al., 


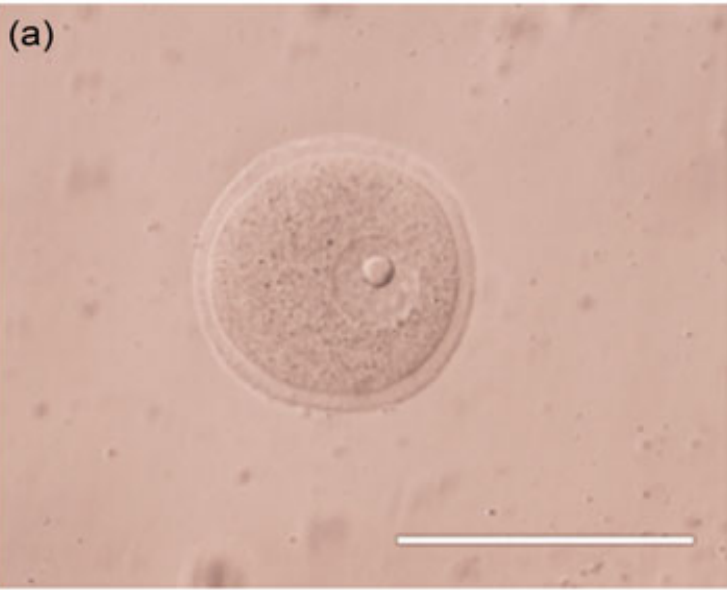

(c)

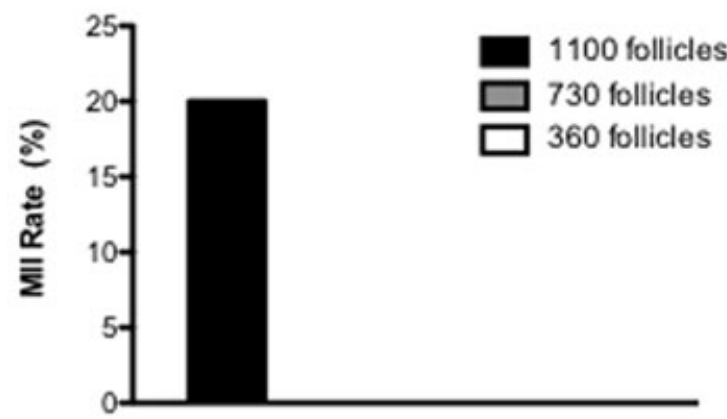

(b)
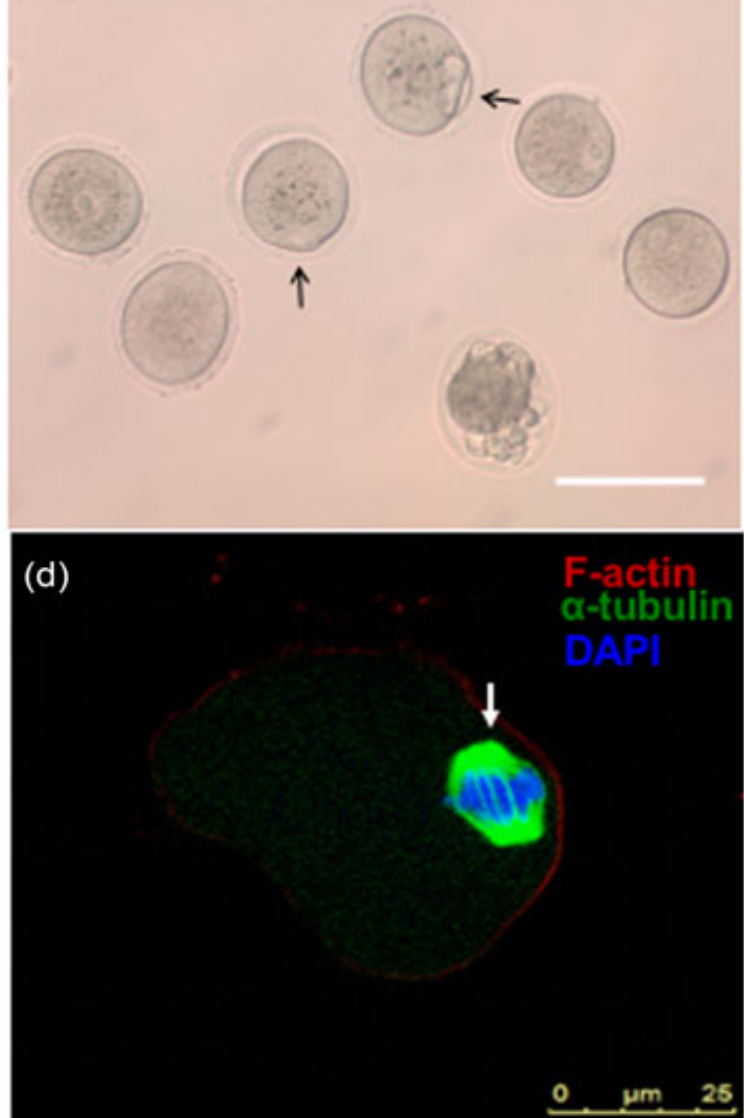

FIGURE 4 Egg retrieval from encapsulated follicles in alginate hydrogels in bursa site 7 Day after transplant and their meiotic maturation in vitro. (a) GV oocytes were retrieved from antral follicles. (b) MII oocytes were confirmed via polar body extrusion (indicated with black arrows). (c) Egg retrieval and MII status from the bursa site. Of the 15 GV oocytes collected at Day 7 from one trial, 3 were MII after IVM (20\% MII rate). Note: MII follicles were only observed with the 1,100-follicle condition and not for the 730- or 360-follicle condition. (d) Normal spindle morphology (indicated by an arrow) was also confirmed in MII oocytes. Image (d) depicts an oocyte obtained from an explant containing ovarian follicles from three ovaries. Scale bar: $100 \mu \mathrm{m}$ (a, b). DAPI, 4',6-diamidino-2-phenylindole; GV, germinal vesicle; IVM, in vitro maturation, MII, metaphase II [Color figure can be viewed at wileyonlinelibrary.com]

2012; Kniazeva et al., 2015; Kreeger, Deck, Woodruff, \& Shea, 2006; Laronda et al., 2014; West, Xu, Woodruff, \& Shea, 2007; Xiao, Duncan et al., 2015; Xiao, Zhang et al., 2015; Xu et al., 2009) and for cell transplantation, such as encapsulated islets as a therapy for type 1 diabetes (Köllmer, Appel, Somo, \& Brey, 2015; Ludwig et al., 2013; Qi, 2014; Scharp \& Marchetti, 2013) and also ovarian follicles (David et al., 2017; Vanacker, Dolmans, Luyckx, Donnez, \& Amorim, 2014). Islets are encapsulated within the hydrogel for isolation from the host over several months, yet the islets are able to survive, sense blood glucose levels, and secrete insulin that can distribute systemically to normalize blood glucose levels. Herein, we used alginate hydrogels for the transplantation of ovarian follicles with the objectives of sensing the hormonal milieu that can drive follicle development and maturation, yet also isolation of the follicles from the host to facilitate their recovery on maturation and prevent the reseeding of cancer cells that may be present within the donor tissue. For the studies herein, the hydrogel was loaded primarily with early-stage follicles (i.e., primordial and primary follicles); the extracted hydrogel grafts contained follicles at all developmental stages, with a notable number of antral follicles. Of the MII oocytes identified after IVM from matured antral follicles, more than a third progressed to the two-cell embryo stage after either ICSI or standard IVF, which is consistent with results found in the previous fertilization studies (Ellenbogen, Shavit, \& Shalom-Paz, 2014; Jin, Lei, Shikanov, Shea, \& Woodruff, 2010; Walls, Junk, Ryan, \& Hart, 2018). These findings confirm the feasibility for use and utility of alginate hydrogels for in vivo maturation of ovarian follicles to obtain meiotically competent oocytes.

Subcutaneous implantation of the hydrogel grafts was initially investigated based on the potential for relatively easy implantation and retrieval, yet the number of follicles recovered was significantly enhanced relative to implantation in the bursa. The bursa site was used as a control for comparison at the subcutaneous site based on the previous reports of live births achieved with transplantation of ovarian tissue and ovarian follicles (Kniazeva et al., 2015; Shikanov, Zhang et al., 2011). Although antral follicles could be recovered from alginate hydrogels implanted into the bursa, and oocyte maturation produced 

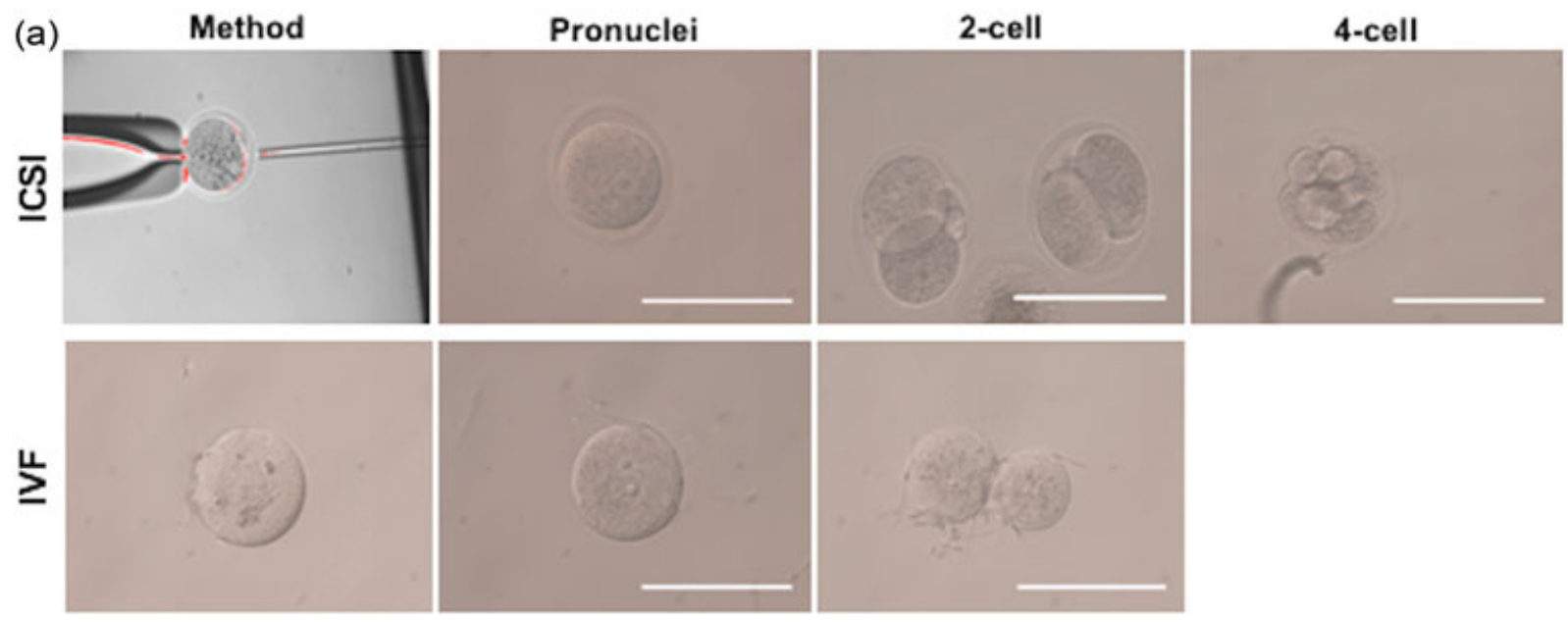

(b)

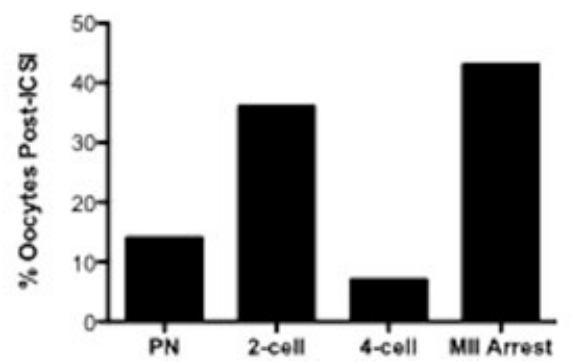

(c)

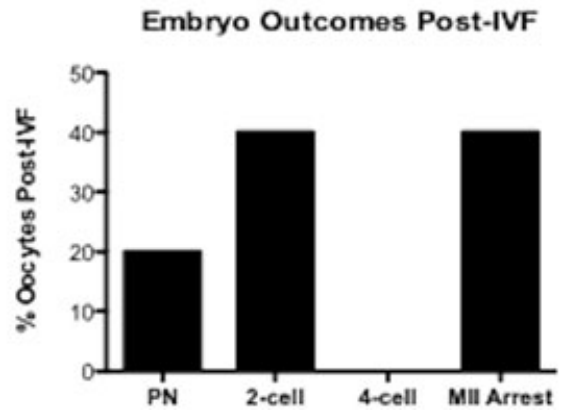

FIGURE 5 Fertilization competency of MII oocytes matured in a subcutaneous site is assessed via ICSI and IVF. (a) Ater ICSI, MII oocytes progressed to the pronucleus, two-cell, and four-cell embryonic stages. After IVF, denuded eggs progressed to the pronucleus and two-cell stages. Scale bar: $100 \mu \mathrm{m}$. (b) After 14 MII oocytes were injected with sperm via ICSI, embryos progressed to the pronucleus (14\%), two-cell (36\%), and four-cell (7\%) stages, whereas $43 \%$ of oocytes remained in MII arrest. (c) Embryos resulting from 10 MII oocytes denuded and placed in an IVF dish with sperm, progressed to the pronucleus (20\%) and two-cell (40\%) stages, whereas $40 \%$ of oocytes remained in MII arrest. Results obtained from two ICSI trials and one IVF trial. ICSI, intracytoplasmic sperm injection; IVF, in vitro fertilization; MII, metaphase II [Color figure can be viewed at wileyonlinelibrary.com]

MII stage oocytes, the hydrogel retrieval was challenging and reduced the yield of follicles. The retrieval process likely contributed to the relatively low number of retrieved follicles from the bursa relative to the subcutaneous site. An additional advantage of subcutaneous implantation is that follicle growth can easily be monitored via ultrasound (Oktay et al., 2001), and human antral follicles can be removed when they mature (typically $>15 \mathrm{~mm}$ ) for oocyte collection. Previous studies by Oktay et al. (2004) demonstrated that although a four-cell human embryo could be obtained from a subcutaneous transplant of ovarian tissue, it failed to implant. To date, only two live human births have been achieved worldwide for transplantation of ovarian tissue at this location (Salama \& Woodruff, 2015). The transplantation of ovarian follicles, rather than ovarian tissue, may enhance the development of the follicles to improve oocyte quality.

Murine oocytes from extracted alginate hydrogels in the subcutaneous site matured to MII could be fertilized and developed to the fourcell embryo stage. The duration of in vivo implantation (7 days) suggests that the antral follicles developed from secondary follicles. Longer transplantation times, approximately 3-7 weeks, are necessary for primordial follicles to develop to the antral stage (Zheng et al., 2014). Collectively, the primordial and primary follicles constitute the most abundant populations in the ovarian reserve. The ability to mature these populations for oocyte collection would ultimately provide options for fertility preservation (Laronda et al., 2014). Future studies may explore the use of exogenous gonadotropins to promote ovarian follicle maturation and increase the number of oocytes retrieved for fertilization (Drummond, 2006; Yang et al., 2006). For either ICSI or standard IVF, $40 \%$ of MII oocytes remained in MII arrest (i.e., did not progress). This result may suggest that oocyte quality can be improved to facilitate progression to a blastocyst stage for embryo transfer. Oocyte quality may be improved by longer maturation periods (>7 days), or modulation of the hydrogel (e.g., alginate and fibrin; Papavasiliou, Sokic, \& Turturro, 2012; Shikanov, Smith, Xu, Woodruff, \& Shea, 2011) or transplant environment. Heterotopic sites offer advantages regarding ease of access, yet may lack the natural cues present at orthotopic sites. Localized delivery of exogenous growth factors, hormones, antioxidants, or pharmacological agents [e.g., phosphatase and tensin homolog (PTEN) inhibitors] provides a means to locally modulate the transplant environment to improve follicle viability and oocyte output after transplant (Demeestere et al., 2009). Gonadotropin treatment before transplant has been shown to improve follicle survival after transplant (Imthurn, Cox, Jenkin, Trounson, \& Shaw, 2000). Such modulation may 


\section{Day 7 Post-Transplant}

\section{Weeks Post-Removal}

(a)

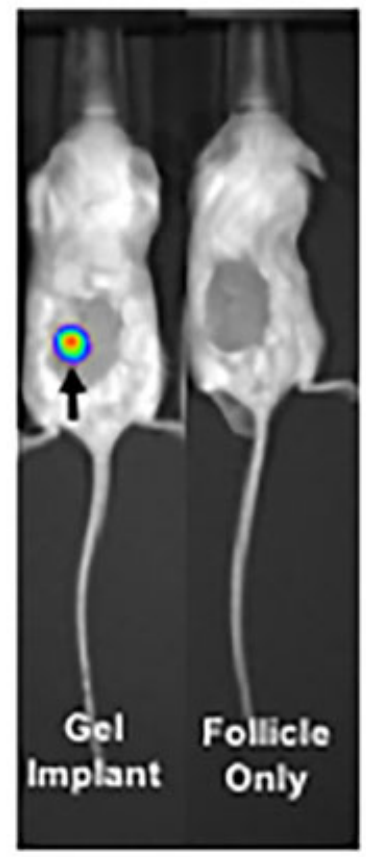

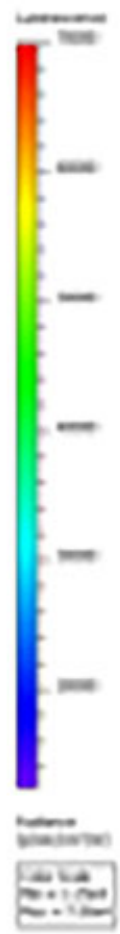

(b)

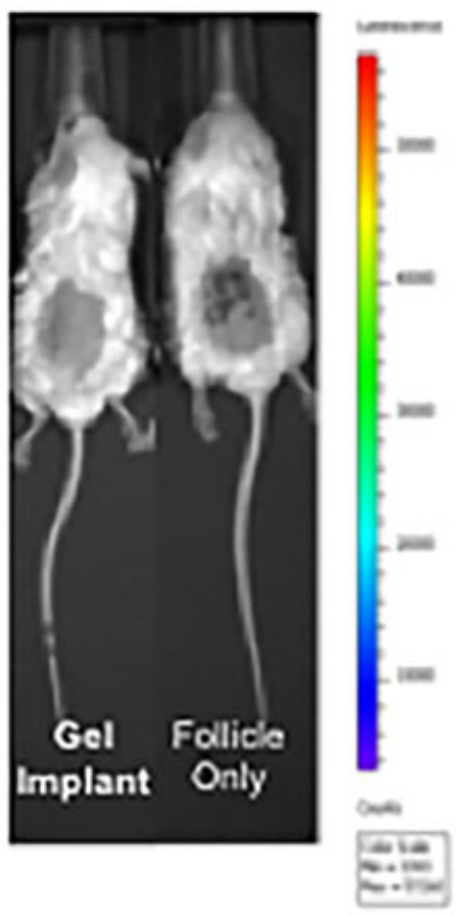

FIGURE 6 In vivo imaging of NSG mice before and after removal of cancer-laden alginate hydrogels. (a) Representative image of an NSG mouse with a subcutaneously transplanted alginate hydrogel containing 200 MDA-MB-231 cells expressing luciferase (imaged Day 7 after transplant). The presence of cancer cells in the hydrogels was confirmed by the positive luminescent signal (signal detection is 600-60,000 counts). The hydrogel implant at Day 7 is denoted with a black arrow. For the control group, mice transplanted with only ovarian follicles were imaged. Cancer cells were present only in the gel implant. (b) Mice were imaged 3 weeks after removal of hydrogels to assess the cancer cell presence. Cancer cells were not detected in experimental mice that had cancer-laden hydrogels removed at Day 7. Cancer cells were not detected in negative controls as well. Three hundred and sixty ovarian follicles were also incorporated into transplanted alginate hydrogels. $n=4$ per group. MDA-MB-231, triple negative breast cancer cell line; NSG, non-obese diabetic-severe combined immunodeficiency (NOD-SCID) gamma [Color figure can be viewed at wileyonlinelibrary.com]

enhance new blood vessel formation around the bead and can be triggered mechanically (Demeestere et al., 2006) or through the delivery of angiogenic factors (e.g., VEGF; Kniazeva et al., 2015; Shikanov, Zhang et al., 2011). Gonadotropin delivery can also upregulate VEGF to induce vessel formation (Demeestere et al., 2009). Taken together, modification to the hydrogel or subcutaneous site may ultimately improve oocyte quality and thus clinical outcomes.

Cryopreserved ovarian tissue from young patients with cancer can contain tens to hundreds of thousands of follicles depending on the age of the patient, and advances in the cryopreservation approach have supported long-term storage of ovarian tissue. One report has confirmed normal tissue morphology for up to 18 years after cryopreservation (Fabbri et al., 2016). Ovarian tissue is typically dissected into relatively small pieces that can be more readily cryopreserved relative to large ovarian tissue chunks. The dissection of ovarian follicles from the tissue can be challenging, owing to the relative dense extracellular matrix of the ovary. Dissecting the ovarian pieces results in retrieval of modest numbers of ovarian follicles (Telfer \& Zelinski, 2013), and thus the fertility preservation strategy must efficiently support survival and maturation given the low numbers of available follicles. The average number of follicles transplanted herein ranged from 360 to 1,100 follicles, with the lowest number of follicles transplanted producing the greatest efficiency for maturating to the antral stage. Additional reductions in follicle number may be necessary for translation.

We demonstrated that the in vivo maturation strategy involving an alginate hydrogel, which does not permit integration of host tissue with the graft, can prevent the escape of cancer cells into vital organs, such as the liver and lungs. The cryopreserved tissue may contain cancer cells, such as those present within the circulation (i.e., circulating tumor cells). In the particular case of leukemia, more than $50 \%$ of cryopreserved ovarian tissue can contain leukemic cells (Soares et al., 2015). The reseeding of disease has been observed clinically with ovarian tissue transplantation (Dolmans et al., 2013). Protocols are being developed to rid ovarian tissue of cancerous cells (Soares et al., 2015) but have not been widely adopted for clinical use, which underscores the need to develop technologies that prevent reseeding of cancer cells. Isolation of transplanted follicles from host tissue has the potential to substantially reduce the number of cancer cells in the graft (Donnez et al., 2011; Kniazeva et al., 2015), yet follicle isolation may not be able to remove the presence of all tumor cells in every graft. Herein, the hydrogel provided 

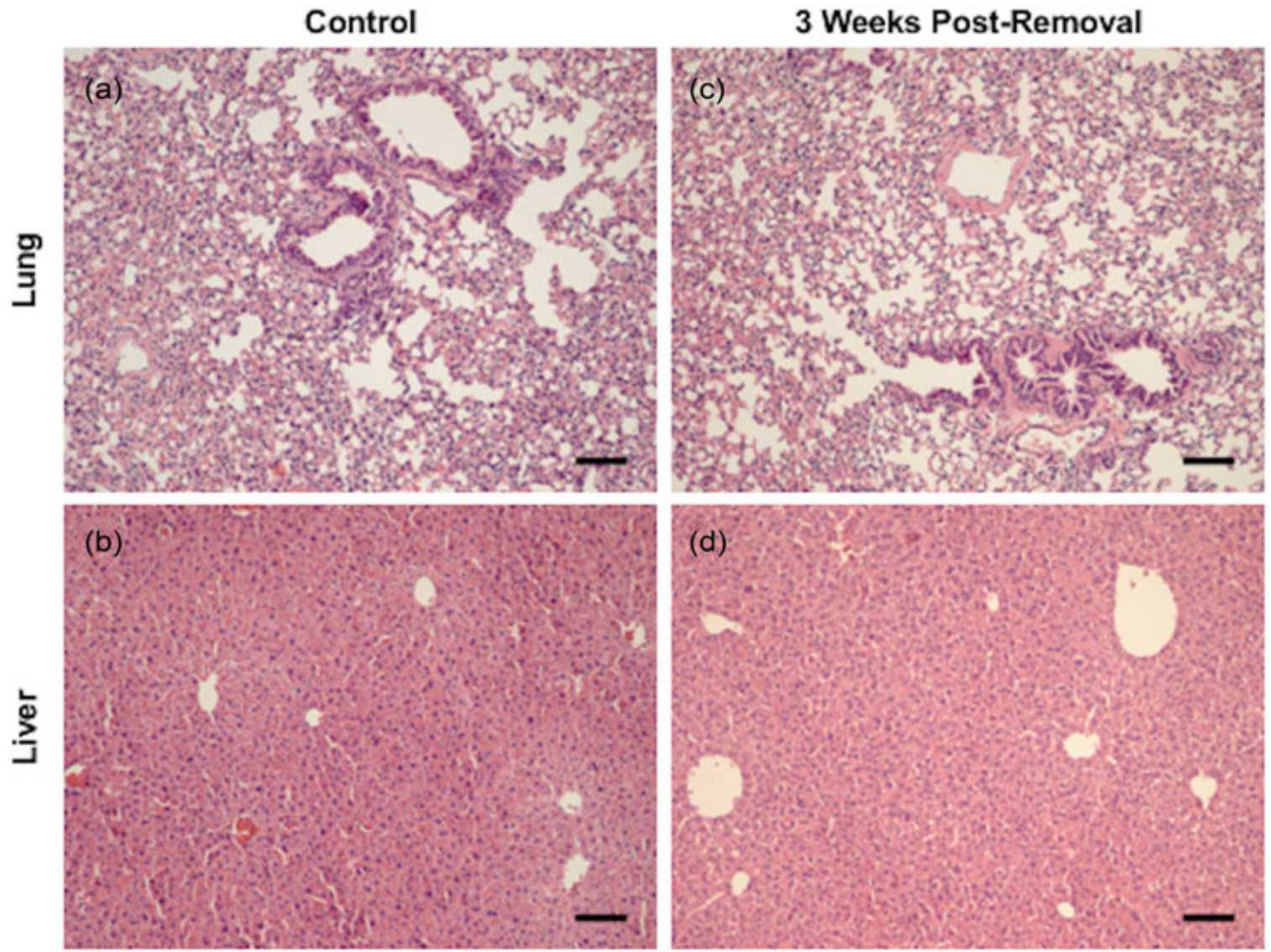

FIGURE 7 Absence of metastatic lesions in liver and lungs of recipient mice 3 weeks after removal of hydrogel. Representative image of (a) liver from naïve NSG control $(n=3)$, (b) lung from naïve NSG control $(n=3)$, (c) liver from a recipient mouse with 200 cancer cells in the hydrogel $(n=4)$, and (d) lung from a recipient mouse with 200 cancer cells in hydrogel $(n=4)$. Lung and liver tissue removed at 3 weeks did not contain any cellular abnormalities or metastatic growths according to staining with H\&E. Scale bar: $100 \mu \mathrm{m}$. H\&E, hematoxylin and eosin; NSG, non-obese diabetic-severe combined immunodeficiency (NOD-SCID) gamma [Color figure can be viewed at wileyonlinelibrary.com]

an additional safety precaution by preventing direct contact of the host tissue with the graft, and it did not allow the escape of the tumor cells to colonize alternative tissues. Previous studies with the transplantation of ovarian tissue indicated that alginate may lose integrity with the expansion of many follicles growing and maturing simultaneously (David et al., 2017). Herein, transplantation was performed with isolated ovarian follicles, which did not allow the escape of cancer, yet the studies were performed for transplant times of 7 days, which was sufficient for the maturation of murine follicles and to confirm the feasibility of the approach. The translation of this strategy to humans may require substantially longer periods of time and development of alternative materials with tunable degradation and mechanical properties to maximize oocyte maturation and recovery.

\section{5 | CONCLUSION}

We present an alginate hydrogel as a retrievable technology to mature ovarian follicles subcutaneously and to prevent escape and subsequent metastasis of cancer cells. Early-stage follicles were transplanted within alginate hydrogels, resulting in retrieval of antral follicles and subsequent collection of oocytes. After IVM, MII oocytes were fertilized and progressed to the two-cell and four-cell embryo stages. These findings collectively demonstrate retrievable hydrogels as a novel approach to mature ovarian follicles to obtain fertilizable oocytes, and also prevent direct contact with host tissue to alleviate concerns related to reseeding disease from cryopreserved autotransplanted ovarian tissue. This strategy may provide a method to enhance safety and improve oocyte quality relative to ovarian tissue transplantation with the potential to improve clinical outcomes for female cancer patients aiming to preserve their fertility.

\section{ACKNOWLEDGMENTS}

This work was supported by the Center for Reproductive Health After Disease (P50HD076188) from the National Institutes of Health National Center for Translational Research in Reproduction and Infertility (NCTRI). 


\section{CONFLICTS OF INTEREST}

The authors declare no conflict of interest.

\section{ORCID}

Lonnie D. Shea (D) http://orcid.org/0000-0002-9296-9673

\section{REFERENCES}

Abir, R., Aviram, A., Feinmesser, M., Stein, J., Yaniv, I., Parnes, D., ... Fisch, B. (2014). Ovarian minimal residual disease in chronic myeloid leukaemia. Reproductive Biomedicine Online, 28, 255-260.

American Cancer Society (2014). Cancer Treatment \& Survivorship Facts \& Figures 2014-2015. Atlanta, GA: American Cancer Society.

American Cancer Society (2017). Cancer Facts and Figures. Atlanta, GA: American Cancer Society. https://old.cancer.org/acs/groups/content/ @editorial/documents/document/acspc-048738.pdf.

Brito, I. R., Lima, I. M. T., Xu, M., Shea, L. D., Woodruff, T. K., \& Figueiredo, R. (2014). Three-dimensional systems for in vitro follicular culture: Overview of alginate-based matrices. Reproduction, Fertility, and Development, 26, 915-930.

Camboni, A, Van Langendonckt, A., Donnez, J., Vanacker, J., Dolmans, M. M., \& Amorim, C. A (2013). Alginate beads as a tool to handle, cryopreserve and culture isolated human primordial/primary follicles. Cryobiology, 67, 64-69.

David, A., Day, J. R., Cichon, A. L., Lefferts, A., Cascalho, M., \& Shikanov, A. (2017). Restoring ovarian endocrine function with encapsulated ovarian allograft in immune competent mice. Annals of Biomedical Engineering, 45, 1685-1696.

Demeestere, I., Simon, P., Emiliani, S., Delbaere, A, \& Englert, Y. (2009). Orthotopic and heterotopic ovarian tissue transplantation. Human Reproduction Update, 15, 649-665.

Demeestere, I., Simon, P., Buxant, F., Robin, V., Fernandez, S. A., Centner, J., ... Englert, Y. (2006). Ovarian function and spontaneous pregnancy after combined heterotopic and orthotopic cryopreserved ovarian tissue transplantation in a patient previously treated with bone marrow transplantation: Case report. Human Reproduction, 21, 2010-2014.

Dolmans, M.-M., Luyckx, V., Donnez, J., Andersen, C. Y., \& Greve, T. (2013). Risk of transferring malignant cells with transplanted frozenthawed ovarian tissue. Fertility and Sterility, 99, 1514-1522.

Donnez, J., \& Dolmans, M.-M. (2015). Ovarian cortex transplantation: 60 reported live births brings the success and worldwide expansion of the technique towards routine clinical practice. Journal of Assisted Reproduction and Genetics, 32, 1167-1170.

Donnez, J., Dolmans, M. -M., Pellicer, A., Diaz-Garcia, C., Sanchez Serrano, M., Schmidt, K. T., ... Andersen, C. Y. (2013). Restoration of ovarian activity and pregnancy after transplantation of cryopreserved ovarian tissue: A review of 60 cases of reimplantation. Fertility and Sterility, 99, 1503-1513.

Donnez, J., Squifflet, J., \& Dolmans, M.-M. (2009). Frozen-thawed ovarian tissue retransplants. Seminars in Reproductive Medicine, 27, 472-478.

Donnez, J., Squifflet, J., Jadoul, P., Demylle, D., Cheron, A. C., Van Langendonckt, A., \& Dolmans, M. M. (2011). Pregnancy and live birth after autotransplantation of frozen-thawed ovarian tissue in a patient with metastatic disease undergoing chemotherapy and hematopoietic stem cell transplantation. Fertility and Sterility, 95, 36-39.

Drummond, A. E. (2006). The role of steroids in follicular growth. Reproductive Biology and Endocrinology, 4, 16. http://www.ncbi.nlm.nih. gov/pmc/articles/PMC1459164/.

Ellenbogen, A., Shavit, T., \& Shalom-Paz, E. (2014). IVM results are comparable and may have advantages over standard IVF. Facts, Views Vision in ObGyn, 6, 77-80.
Fabbri, R., Macciocca, M., Vicenti, R., Pasquinelli, G., Caprara, G. Valente, S., ... Paradisi, R. (2016). Long-term storage does not impact the quality of cryopreserved human ovarian tissue. Journal of Ovarian Research, 9, 1-10.

Filatov, M. A., Khramova, Y. V., \& Semenova, M. L. (2014). In vitro mouse ovarian follicle growth and maturation in alginate hydrogel: Current state of the art. Acta Naturae, 7, 48-56.

Hornick, J. E., Duncan, F. E., Shea, L. D., \& Woodruff, T. K. (2012). Isolated primate primordial follicles require a rigid physical environment to survive and grow in vitro. Human Reproduction, 27, 1801-1810.

Hornick, J. E., Duncan, F. E., Shea, L. D., \& Woodruff, T. K. (2013). Multiple follicle culture supports primary follicle growth through paracrineacting signals. Reproduction, 145, 19-32.

Imbert, R., Moffa, F., Tsepelidis, S., Simon, P., Delbaere, A., Devreker, F., ... Demeestere, I. (2014). Safety and usefulness of cryopreservation of ovarian tissue to preserve fertility: A 12-year retrospective analysis. Human Reproduction, 29, 1931-1940.

Imthurn, B., Cox, S. L., Jenkin, G., Trounson, A. O., \& Shaw, J. M. (2000). Gonadotrophin administration can benefit ovarian tissue grafted to the body wall: Implications for human ovarian grafting. Molecular and Cellular Endocrinology, 163, 141-146.

Jin, S. Y., Lei, L., Shikanov, A., Shea, L. D., \& Woodruff, T. K. (2010). A novel two-step strategy for in vitro culture of early-stage ovarian follicles in the mouse. Fertility and Sterility, 93, 2633-2639.

Kim, S. Y., Kim, S. K., Lee, J. R., \& Woodruff, T. K. (2016). Toward precision medicine for preserving fertility in cancer patients: Existing and emerging fertility preservation options for women. Journal of Gynecologic Oncology, 27, e22. http://www.pubmedcentral.nih.gov/ articlerender.fcgi?artid=4717227\&tool=pmcentrez\&rendertype $=$ abstract.

Kniazeva, E., Hardy, A. N., Boukaidi, S. A., Woodruff, T. K., Jeruss, J. S., \& Shea, L. D. (2015). Primordial follicle transplantation within designer biomaterial grafts produce live births in a mouse infertility model. Scientific Reports, 5, 17709. http://www.pubmedcentral.nih.gov/ articlerender.fcgi?artid=4668556\&tool=pmcentrez\&rendertype= abstract.

Köllmer, M., Appel, A. A., Somo, S. I., \& Brey, E. M. (2015). Long-term function of alginate-encapsulated islets. Tissue Engineering. Part B, Reviews, 22, 34-46. http://www.ncbi.nlm.nih.gov/pubmed/26414084.

Kondapalli, L. A., \& Ginsberg, J. P. (2012). Ovarian tissue cryopreservation and transplantation, Oncofertility Medical Practice: Clinical Issues and Implementation. 63-75. New York, NY: Springer Science +Business Media. http://oncofertility.northwestern.edu/sites/ oncofertility/files/legacy_files/uploadedfilecontent/ovarian_tissue_ cryopreservation_and_transplantation_-_laxmi_a._kondapalli.pdf.

Kreeger, P. K., Deck, J. W., Woodruff, T. K., \& Shea, L. D. (2006). The in vitro regulation of ovarian follicle development using alginateextracellular matrix gels. Biomaterials, 27, 714-723.

Laronda, M. M., Duncan, F. E., Hornick, J. E., Xu, M., Pahnke, J. E., Whelan, K. A., ... Woodruff, T. K. (2014). Alginate encapsulation supports the growth and differentiation of human primordial follicles within ovarian cortical tissue. Journal of Assisted Reproduction and Genetics, 31, 1013-1028.

Ludwig, B., Reichel, A., Steffen, A., Zimerman, B., Schally, A. V., Block, N. L., ... Bornstein, S. R. (2013). Transplantation of human islets without immunosuppression. Proceedings of the National Academy of Sciences of the United States of America, 110, 1-5.

Luyckx, V., Dolmans, M.-M., Vanacker, J., Legat, C., Fortuño Moya, C., Donnez, J., \& Amorim, C. A. (2014). A new step toward the artificial ovary: Survival and proliferation of isolated murine follicles after autologous transplantation in a fibrin scaffold. Fertility and Sterility, 101, 1149-1156.

Meirow, D., Ra, H., \& Biderman, H. (2014). Ovarian tissue cryopreservation and transplantation: A realistic, effective technology for fertility preservation. Human fertility, methods and protocols (p. 1154). New 
York, NY: Humana Press. http://link.springer.com/10.1007/978-14939-0659-8.

Oktay, K., Economos, K., Kan, M., Rucinski, J., Veeck, L., \& Rosenwaks, Z. (2001). Endocrine function and oocyte retrieval after autologous transplantation of ovarian cortical strips to the forearm. The Journal of the American Medical Association, 286, 1490-1493.

Oktay, K., Buyuk, E., Veeck, L., Zaninovic, N., Xu, K., Takeuchi, T., ... Rosenwaks, Z. (2004). Embryo development after heterotopic transplantation of cryopreserved ovarian tissue. Lancet, 363, 837-840.

Papavasiliou, G., Sokic, S., \& Turturro, M. (2012). Synthetic PEG hydrogels as extracellular matrix mimics for tissue engineering applications, Biotechnology: Molecular Studies and Novel Applications for Improved Quality of Human Life (111-135). London, United Kingdom: InTech Open.

Qi, M. (2014). Transplantation of encapsulated pancreatic islets as a treatment for patients with type 1 diabetes mellitus. Advances in Medicine, 2014, 1-15.

Rosendahl, M., Greve, T., \& Andersen, C. Y. (2013). The safety of transplanting cryopreserved ovarian tissue in cancer patients: A review of the literature. Journal of Assisted Reproduction and Genetics, 30, 11-24.

Salama, M., \& Woodruff, T. K. (2015). New advances in ovarian autotransplantation to restore fertility in cancer patients. Cancer Metastasis Reviews, 34, 807-822.

Scharp, D. W., \& Marchetti, P. (2013). Encapsulated islets for diabetes therapy: History, current progress, and critical issues requiring solution. Advanced Drug Delivery Reviews, 67-68, 35-73.

Shea, L. D., Woodruff, T. K., \& Shikanov, A. (2014). Bioengineering the ovarian follicle microenvironment. Annual Review of Biomedical Engineering, 16, 29-52.

Shikanov, A., Ph, D., Zhang, Z., Xu, M., Smith, R. M., Rajan, A., ... Shea, L. D. (2011). Fibrin encapsulation and vascular endothelial growth factor delivery promotes ovarian graft survival in mice. Tissue Engineering. Part A, 17, 3095-3104.

Shikanov, A., Smith, R. M., Xu, M., Woodruff, T. K., \& Shea, L. D. (2011). Hydrogel network design using multifunctional macromers to coordinate tissue maturation in ovarian follicle culture. Biomaterials, 32, 2524-2531.

Shikanov, A., Zhang, Z., Xu, M., Smith, R. M., Rajan, A., Woodruff, T. K., \& Shea, L. D. (2011). Fibrin encapsulation and vascular endothelial growth factor delivery promotes ovarian graft survival in mice. Tissue Engineering. Part A, 17, 3095-3104.

Smith, R. M., Shikanov, A., Kniazeva, E., Ramadurai, D., Woodruff, T. K., \& Shea, L. D. (2014). Fibrin-mediated delivery of an ovarian follicle pool in a mouse model of infertility. Tissue Engineering. Part A, 20, 3021-3030.

Soares, M., Sahrari, K., Amorim, C. A., Saussoy, P., Donnez, J., \& Dolmans, M.-M. (2015). Evaluation of a human ovarian follicle isolation technique to obtain disease-free follicle suspensions before safely grafting to cancer patients. Fertility and Sterility, 104, 672-680. e2.

Telfer, E. E., \& Zelinski, M. B. (2013). Ovarian follicle culture: Advances and challenges for human and nonhuman primates. Fertility and Sterility, 99, 1523-1533.

Vanacker, J., Dolmans, M.-M., Luyckx, V., Donnez, J., \& Amorim, C. A. (2014). First transplantation of isolated murine follicles in alginate. Regenerative Medicine, 9, 609-619.
Wallace, W. H. B., Kelsey, T. W., \& Anderson, R. A. (2016). Fertility preservation in pre-pubertal girls with cancer: The role of ovarian tissue cryopreservation. Fertility and Sterility, 105, 6-12.

Wallace, W. H. B., Shalet, S. M., Hendry, J. H., Morris-Jones, P. H., \& Gattamaneni, H. R. (1989). Ovarian failure following abdominal irradiation in childhood: The radiosensitivity of the human oocyte. The British Journal of Radiology, 62, 995-998.

Walls, M., Junk, S., Ryan, J. P., \& Hart, R. (2018). IVF versus ICSI for the fertilization of in-vitro matured human oocytes. Reproductive Biomedicine Online, 25, 603-607.

West, E., Xu, M., Woodruff, T., \& Shea, L. (2007). Physical properties of alginate hydrogels and their effects on in vitro follicle development. Biomaterials, 28, 4439-4448.

Wo, J. Y., \& Viswanathan, A. N. (2009). Impact of radiotherapy on fertility, pregnancy, and neonatal outcomes in female cancer patients. International Journal of Radiation Oncology, Biology, Physics, 73, 1304-1312.

Xiao, S., Duncan, F. E., Bai, L., Nguyen, C. T., Shea, L. D., \& Woodruff, T. K. (2015). Size-specific follicle selection improves mouse oocyte reproductive outcomes. Reproduction, 150, 183-192.

Xiao, S., Zhang, J., Romero, M. M., Smith, K. N., Shea, L. D., \& Woodruff, T. K. (2015). In vitro follicle growth supports human oocyte meiotic maturation. Scientific Reports, 5, 17323. http://www.pubmedcentral.nih. gov/articlerender.fcgi?artid=4661442\&tool=pmcentrez\&rendertype $=$ abstract.

Xu, M., Banc, A., Woodruff, T. K., \& Shea, L. D. (2009). Secondary follicle growth and oocyte maturation by culture in alginate hydrogel following cryopreservation of the ovary or individual follicles. Biotechnology and Bioengineering, 103, 378-386.

Xu, M., Kreeger, P. K., Shea, L. D., \& Woodruff, T. K. (2006). Tissueengineered follicles produce live, fertile offspring. Tissue Engineering, 12, 2739-2746.

Yang, H. Y., Cox, S. -L., Jenkin, G., Findlay, J., Trounson, A., \& Shaw, J. (2006). Graft site and gonadotrophin stimulation influences the number and quality of oocytes from murine ovarian tissue grafts. Reproduction, 131, 851-859.

Zheng, W., Zhang, H., Gorre, N., Risal, S., Shen, Y., \& Liu, K. (2014). Two classes of ovarian primordial follicles exhibit distinct developmental dynamics and physiological functions. Human Molecular Genetics, 23, 920-928.

\section{SUPPORTING INFORMATION}

Additional supporting information may be found online in the Supporting Information section at the end of the article.

How to cite this article: Rios PD, Kniazeva E, Lee HC, et al. Retrievable hydrogels for ovarian follicle transplantation and oocyte collection. Biotechnology and Bioengineering. 2018;115: 2075-2086. https://doi.org/10.1002/bit.26721 